\title{
PERFORMANCE OF THE NEW NEURAL NETWORK BASED CONTROL STRUCTURE AND LEARNING ALGORITHM
}

\author{
VOJSLAV KECMAN ${ }^{1}$ and LJUBIŠA VLAČIC ${ }^{2}$ \\ ${ }^{1}$ The University of Auckland, Department of Mechanical Engineering \\ Private Bag 92019, Auckland, New Zealand, v.kecman@auckland.ac.nz \\ ${ }^{2}$ Griffith University, School of Microelectronic Engineering, \\ Nathan Campus TB 3.10, Brisbane, Australia, L.Vlacic@me.gu.edu.au
}

\begin{abstract}
This paper presents a new neural network (NN) based Adaptive Backthrough Control ( $A B C)$ scheme for both linear and nonlinear dynamic plants. A feedforwand approach presented here falls into the direct design category. In its simplest form the implementation requires an estimation of the process parameters at any sample instant. Unlike the other feedforward NN based control schemes the $A B C$ proposed here comprises of one neural network only which simultaneously acts as both - plant model (emulator) and the controller (inverse of the emulator). For linear plants, without noise, the resulting feedforward controller, providing that the order of the plant and plant model are equal, is a perfect adaptive poleszeros canceller. In the case of nonlinear dynamic system, and for the monotonic nonlinearity, the proposed $\mathrm{ABC}$ control represents the nonlinear predictive controller. The ABC scheme is based on the discrete nonlinear (NARMAX) dynamic model. For such models and for monotonic nonlinearity, the calculation of the desired control signal is the result of the nonlinear optimization procedure with guaranteed convex search function and consequently with an unique solution.
\end{abstract}

Key words: Neuro-control, nonlinear adaptive control

\section{Introduction and Basic Control Structure}

This paper focuses on the $\mathrm{NN}$ based adaptive control. Due to the 'equality' of NN and fuzzy logic (FL) models (see Kecman and Pfeiffer, 1994) the same approach can be applied to the adaptive FL based control schemes. In particular, after presenting the basic guiding ideas of the $\mathrm{NN}$ based control approaches, we will introduce the Adaptive Backthrough Control $(A B C)$ scheme as one of the most serious candidate for the future control of the large class of nonlinear, partially known and timevarying systems [Kecman, 1997; Kecman and Rommel, 1997; Rommel, 1997, Salman and Kecman, 1998]. Recently, the area of NN control has been exhaustively investigated and there is a large number of different NN based control methods. A systematic classification of the very different $\mathrm{NN}$ control structures is a formidable task indeed [Agarwal, 1997].

So-called 'standard' or 'classic' NN based control uses two neural networks. This control structure comprises $\mathrm{NN}_{2}$ which represents the (approximate) model of the plant, and $\mathrm{NN}_{1}$ that functions as a controller. The latter one represents (approximate, again) inverse of $\mathrm{NN}_{2}$. Note that $\mathrm{NN}_{1}$ is an 'inverse' of the plant model and not of the plant.

Here proposed Adaptive Backthrough Control $(A B C)$, is in the spirit of the basic results and approaches from [Psaltis et al, 1988; Saerens and Soquet, 1991; Garcia and Morari, 1982; Jordan, 1993; Hunt and Sbarbaro, 1991; Narendra and Parthasarathy, 1990 and Widrow and Walach, 1996]. While being 'similar' in spirit, there are few important distinctive features which differ the $\mathrm{ABC}$ approach from all the other standard NN based control methods.

First of all, the ABC uses one neural network only [Kecman, 1997, Salman and Kecman, 1998]. Second principal feature of the $A B C$ is that, unlike the other approaches, it doesn't use standard training errors as the learning signals for modeling the plant and adapting the controller. (Note that at the $\mathrm{ABC}$ approach, the $\mathrm{NN}$ is both the plant model and the controller). Rather, the true desired value $y_{d}$ (signal that should be tracked, reference signal) is used for the training of the NN. In this way, the desired but unknown control signal $u_{d}$, results from the backward transformation of the $y_{d}$ through the $\mathrm{NN}$. The origin of the label for this approach as a backthrough method, lies in this backward step for the calculation of $u_{d}$. In this way the ABC basically represents a younger (and it seems more direct and powerful) relative of the 'distal teacher' idea from [Jordan, 1993] or of the [Saerens and Soquet, 1990], as well as of [Saerens, Renders, and Bersini, 1996] approach.

Similarly to the adaptive inverse control (AIC) devised by Widrow, ABC control scheme proposed here is effective as long as the plant is stable. It solves the problems of tracking and disturbance rejection for any stable plant. The same will be true in the case of unstable plants as long as 
the unstable plant is stabilized by some classic control method first. The reference block presented in Fig 1 is not required, unless some control of the control signal variable $u$ is needed. All results below are obtained by using $G_{\text {ref }}(s)=1$.

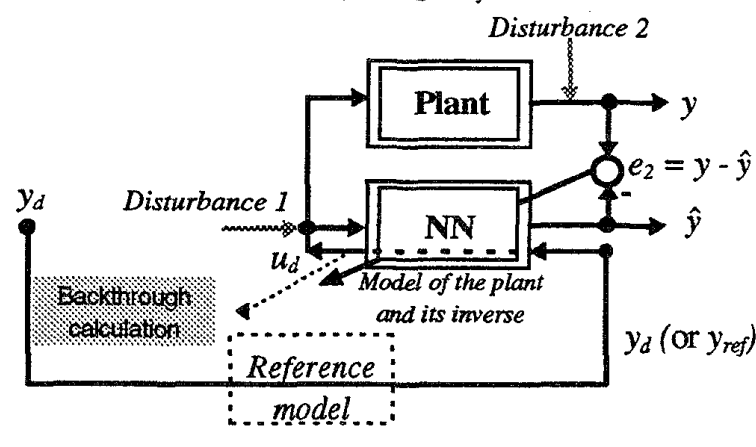

Figure 1 Neural or fuzzy network based Adaptive Backthrough Control ( $A B C$ ) scheme with one network which simultaneously acts as the plant model and as a controller (inverse plant model).

The first papers on the $\mathrm{NN}$ based $\mathrm{ABC}$ system had described the $\mathrm{ABC}$ structure comprised of the two NN [Kecman and Rommel, 1997] and [Rommel, 1997]. This structure was 'inherited' from the previously mentioned approaches and it is directly related to the classic EBP learning. The task of the first network $\mathrm{NN}_{1}$ was to act as a controller i.e., to learn the inverse dynamics of the controlled plant. The second $\mathrm{NN}, \mathrm{NN}_{2}$ was used as the plant model (needed as the part of the EBP procedure for learning of the $\mathrm{NN}_{1}$ 's weights). Being properly trained and after receiving the desired plant output signal $y_{d}$, NN1 was able to produce the best control signal $u_{d}$ which would drive the plant to output the desired $y_{d}$. However, the $\mathrm{ABC}$ learning is different from the EBP algorithm. Note that in the $A B C$ algorithm by using the desired trajectory $y_{d}$, the best control signal $u_{d}$ can be calculated directly by the backward step through the single NN. Thus, having the two NN in the control structure, there is a great deal of a redundancy and it seems as though both the very structure of the whole control system and the learning can be halved. Having the signal $u_{d}$ calculated, the controller network $\mathrm{NN}_{1}$ is no longer needed. The $A B C$ structure with only one NN which simultaneously acts as the plant model and as a controller (inverse plant model) is shown in Fig 1.

The standard control task and the basic problem in controlling an unknown dynamic plant is to find the proper, or desired, control (actuation) value $u_{d}$ as an input to the plant which should ensure that,

$$
y(t)=y_{d}(t), \quad \forall t
$$

where the subscript $d$ stands for desired. The variables $y(t)$ and $y_{d}(t)$ denote the actual plant output and desired (reference) plant output respectively. A controller that could produce this value $u_{d}$ would be the best controller and the output of the plant would exactly follow the desired input $y_{d}$. In linear control, (51) will be ensured when,

$$
G_{c i}(s)=G_{p}^{-1}(s)
$$

Hence, the ideal controller transfer function $G_{c i}(s)$ should be the inverse of the plant transfer function $G_{p}(s)$. Because of many practical constraints, this is an idealized control structure [Kecman, 1988]. However, we can try to get as close as possible to this ideal controller solution $\left(G_{c i}(s)\right)$. The $\mathrm{ABC}$ approach which is presented in this section, can achieve a great deal (sometimes even nearly all) of this ideal controller. The block diagram of the ideal control of any nonlinear system is given in Fig 2.

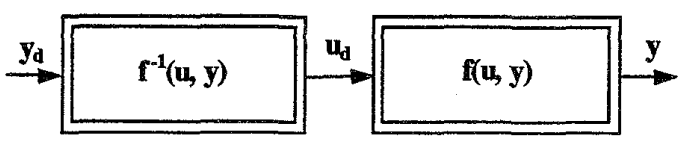

Figure 2 The ideal (feedforward) control structure for any plant.

$f(u, y)$ represented in Fig 2 stands for any nonlinear mapping between an input $\mathbf{u}(t)$ and an output $\mathbf{y}(t)$. In a general case of a dynamic system $f(u, y)$ represents a system of nonlinear differential equations. Here we will primarily be concerned with discrete-time systems, and the model of the plant in the discrete-time domain will be in the form of nonlinear discrete equation $\mathbf{y}(k+I)=$ $\mathbf{f}(\mathbf{u}(k), \mathbf{y}(k))$. Now, the basic problem is how to learn, or obtain, the inverse model of the unknown dynamic plant by using NN?

The wide application of NN in control is based on the universal approximation capacity of neural networks and fuzzy models. Thus a learning (identification, adaptation, training) of the plant and inverse plant dynamics represents both the basic mathematical tool and the basic problem to be solved.

So far as the representation of dynamic system is concerned, we use a so-called NARMAX model here. In the extensive literature on modeling dynamic plants, it was proved that under some mild assumptions any nonlinear, discrete and time invariant system can always be represented by the following NARMAX model,

$$
y(k+1)=f\{y(k), \cdots, y(k-n) ; u(k), \cdots, u(k-m)\},
$$


where $y_{k}$ and $u_{k}$ are the input and output signals at instant $k$, and $y_{k-i}$ and $u_{k-j}(i=1, \ldots, n$ and $j=1, \ldots$ , $m$ ) represent the past values of these signals. Typically one can work with $n=m$. (3) is a simplified deterministic version of the NARMAX model (there is no noise terms in it), and is valid for dynamic systems with $K$ outputs and $L$ inputs. For $K=L=1$ we obtain the so-called SISO (single-input single-output) system which is studied here.

In reality, the nonlinear function $f$ from (3) is very complex and generally unknown. The whole idea in the application of $\mathrm{NN}$ is to try to approximate $f$ by using some known and simple functions which, in the case of the application of NN and FLM, are their activation and membership functions respectively. Both the identification part and the control part in NN can be given a graphical representation (Fig 3). Note that two different identification schemes are presented in Fig 3 - series-parallel and parallel. (The names are due [Landau, 1979]). The identification can be done by using either of the two schemes,

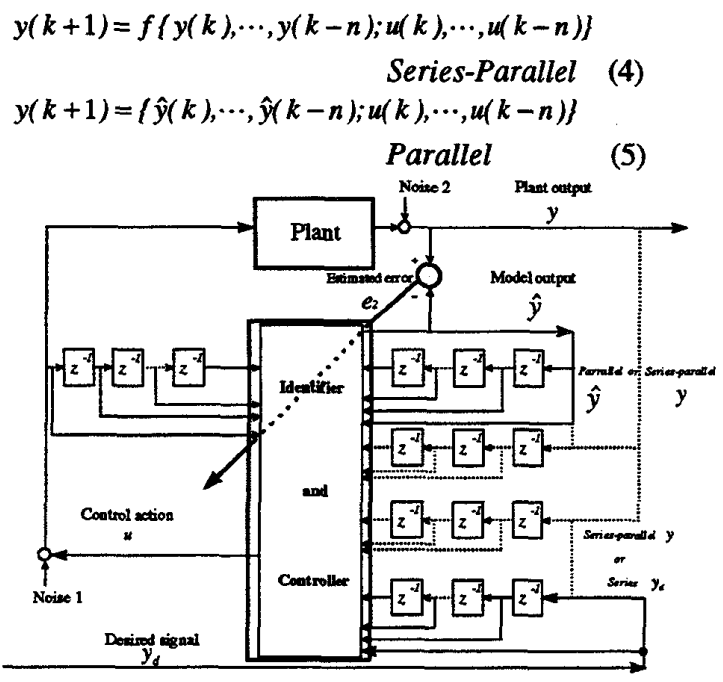

Figure 3 Identification and control scheme using NN.

It is hard to say which identification scheme is better. Narendra and Annaswamy (1989) showed (for linear systems) the series-parallel method to be globally stable. The parallel method has the advantage of avoiding noises existing in real plant output signals. On the other hand series-parallel scheme uses actual (meaning correct) plant outputs and this generally enforces identification. Three are also two control schemes presented in Fig 3: a series-parallel and a series scheme. The detailed analysis of the performance of both schemes, in the case of the linear plant only, is given in [Salman and Kecman, 1998], where it is shown that under some mild assumptions on the identification part, the series-parallel scheme is superior in the linear case.

The determination of a good inverse model can be done by using many different and, more or less suitable, approaches. We will only mention the three most popular ones as presented in [Psaltis et al, 1988]. In their paper they introduced and discussed - a general, indirect and specialized learning architecture for the NN based control of the stable nonlinear plants. (Independently, the same approach as the general architecture was developed in [Jordan and Rumelhart, 1992] and it was named as a direct inverse modeling. This is basically an off-line procedure and for nonlinear plants it will usually precede the on-line phase. (If the plant is unstable a stabilization with a feedback loop is necessary. That can be done with any standard control algorithm). Detailed description of these approaches can be found in [Kecman, 1997]. Here we will present only the basic ideas and the performance of the $A B C$ scheme.

\section{Learning in the $A B C$ Structures Which Comprises One NN Only}

Fig 1 shows the $\mathrm{ABC}$ scheme having one NN which acts as both the plant model (emulator) and the controller of the plant. The most important part of the learning is the calculation of the desired control signal $u_{d}$. This calculation is done in an on-line mode. In the case that the plant is nonlinear the standard approach is that this on-line part is preceded by the off-line learning of the plant model. The advantage is that the off-line learning will produce a better set of initial weights for the on-line operation. In the case of nonlinear plants pretraining of $N N$ is essential. For the linear plant this pretraining is not important.

Sometimes it may be useful to introduce a reference model, too. This step is not crucial for the ABC approach but an important result could be that with a reference model a tuning of the control effort is possible. This might be necessary for many real existing systems because the actuators usually operate only within a specific range, and leaving this range is either not possible or can harm the system's performance.

The basic idea of the $A B C$ is to design a plant emulator which simultaneously acts as the inverse of the plant (or, as an adaptive controller). In this way, the problem of finding the 'best' control signal $u_{d}$ will be solved. In general, this value $u_{d}$ is not available. By using the $\mathrm{ABC}$ 
value $u_{d}$ is not available. By using the $\mathrm{ABC}$ approach we can find this desired control values $u_{d}$ that will usually be very close to the ideal ones.

For the $A B C$ of linear systems, the calculation of $u_{d}$ is straightforward. The forward model (NN in Fig 1 and Fig 3 ) is given as,

$$
\hat{y}(k+1)=\sum_{i=1}^{N} w_{2 ;} \cdot x_{2, i}=w_{2}^{T} \cdot \mathbf{x}_{2}
$$

where $N=2 n, n$ is the order of the model and $x_{2}$ is an input vector to the NN comprised of present and previous values of $u$ and $y$. For the calculation of the desired value $\hat{u}_{d}$ this equation should and can be rearranged in respect to the input of the neural network $\mathrm{NN}_{2}$,

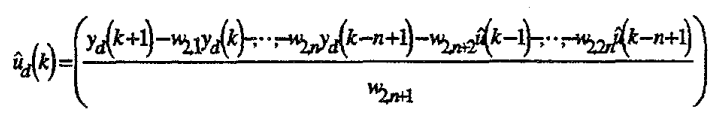

Therefore, when applied to the control of linear systems, the calculation of the control signal $u_{d}$ by using (7) is similar to the predictive (deadbeat) controller approach. Note that in the calculation of the best estimates of desired control signal $\hat{u}_{d}(k)$ to the plant and to the NN, the desired output values of system $y_{d}(k+1), y_{d}(k), \cdots, y_{d}(k-n)$ are used. This is so-called series model presented in Fig 3. It is interesting to note that instead of using the present and previous desired values, one can use the present and previous actual plant outputs $y(k), \cdots, y(k-n)$. This is so-called series-parallel model presented in Fig 3 which is in the case of linear plant superior to the series control model.

In the case of the nonlinear system control, the calculation of the desired control signal $u_{d}$ which corresponds to the desired output from the plant $y_{d}$, is much more involved task. For the monotonic nonlinearities (i.e., for the one-to-one inverse mapping of the plant outputs $y$ into its inputs $u$ ) control signal $u_{d}$ can be calculated by an iterative algorithm which guarantees finding of proper $u_{d}$ for any desired $y_{d}$. This is the crucial result in the proposed $A B C$ algorithm. All the details of the numerical part of the backthrough calculation of $u_{d}$ can be found in [Kecman, 1997]. The two other alternative approaches to the calculation of the $u_{d}$ which deserve more investigations are given in [Rommel, 1997].

\section{Simulational Results}

Example 1: Nonlinear monotonic 1st order dynamic plant adapted from [Narendra and Parthasarathy, 1990] should be controlled by the $\mathrm{ABC}$ scheme comprised of the one network only. The plant equation is given below,

$$
y(k)=\frac{y(k-1)}{1+y^{2}(k-1)}+u^{3}(k-1)
$$

The neural network which simultaneously acts as a plant model and as its controller is comprised of 39 neurons in hidden layer. Basis functions in all $\mathrm{HL}$ neurons are the two-dimensional Gaussians with the same covariance matrix $\Sigma=\operatorname{diag}(0.2750$, 0.0833 ), and with positions determined by an orthogonal least squares selection procedure [Orr, 1996]. NN was pretrained by using 1000 data pairs. Training input signal was a uniformly distributed random signal. (Note that the $A B C$ control structure is much simpler than the one in [Narendra and Parthasarathy, 1990]. They used two NN for the identification and one as a controller. Each network had 200 neurons. Besides, in the off-line training phase they used 25 000 training pairs).

After the training was done, a number of simulation runs had proved very good performance of the $\mathrm{ABC}$ scheme while controlling time invariant nonlinear system. Fig 4 (left) shows the plant response while tracking input $y_{d}=\sin (2 \pi k /$ $25)+\sin (2 \pi k / 10)$. The plant response is indistinguishable from the desired trajectory. The tracking is perfect.

Much more complex task is to control the time variant nonlinear plant. There is no general theory, approach or method in adaptive control of nonlinear time variant plants. These are the toughest control problems anyway. Here, we only present initial results on how the $A B C$ scheme cope with such plants. We do not pretend to answer any open question in this field, but rather we try to put a little light on its performance. Fig 4 (right) shows the error when the pretrained but fixed NN tried to control fast changing plant given below,

$y(k)=\frac{y(k-1)}{1+y^{2}(k-1)}+(1-0.001 k) * u^{3}(k-1)$

This is a model of the plant which halves the plant gain in 500 steps. Without an adaptation the performance error $e_{3}=y_{d}-y$ increases rapidly (Fig 4 , right). Fig 5 shows error in the case of the online adaptation of neural network. Results are obtained by using a forgetting factor $\lambda=0.985$. 

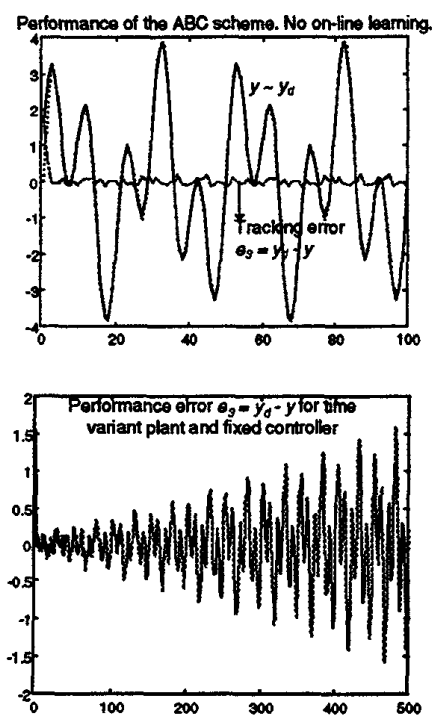

Figure 4 ABC: Perfect tracking in the case of nonlinear monotonic time invariant plant (top). Performance error for fixed pretrained NN controlling the time variant plant (bottom). (The plant gain is halved in 500 steps).
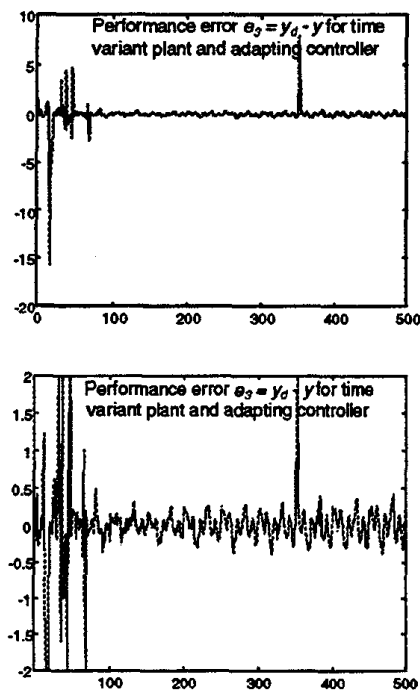

Figure 5 ABC: Performance error at controlling the time variant plant with an on-line adaptation of the NN OL weights. Forgetting factor $\lambda=0.985$. (Bottom graph is drawn in the same scale as Fig 6 bottom).

The adaptation and control process is stable and, in comparison to the error in Fig 4, the final error in Fig 5 is three times smaller.

Example 2: Consider the $\mathrm{ABC}$ of the following nonlinear non-monotonic dynamic plant,

$$
y_{k+1}=\sin \left(y_{k}\right) * \sin \left(u_{k}\right)-u_{k} / \pi
$$

This plant is an non-monotonic nonlinear function. In other words, there is one-to-many mapping of the $u_{k}$ to the $y_{k+1}$. However, the function $y_{k+1}=f$ $\left(u_{k}, y_{k}\right)$ represents an one-to-one mapping and the $\mathrm{ABC}$ can successfully model both the plant dynamics (mapping of $u$ to $y$ ) and the plant inverse dynamics (mapping of $y$ to $u$ ). The $\mathrm{NN}$ was optimized by using a feedforward orthogonal least square method. The basis functions in all neurons are the 2-dimensional Gaussians with the same covariance matrix $\Sigma=\operatorname{diag}(0.0735,0.1815)$. At the beginning of the RBF selection, there were 169 symmetrically placed neurons in hidden layer and at the and 47 centers were chosen. Such a network models the plant very well. (Note that this structure corresponds to the fuzzy logic model with a rule basis comprised of 47 rules).
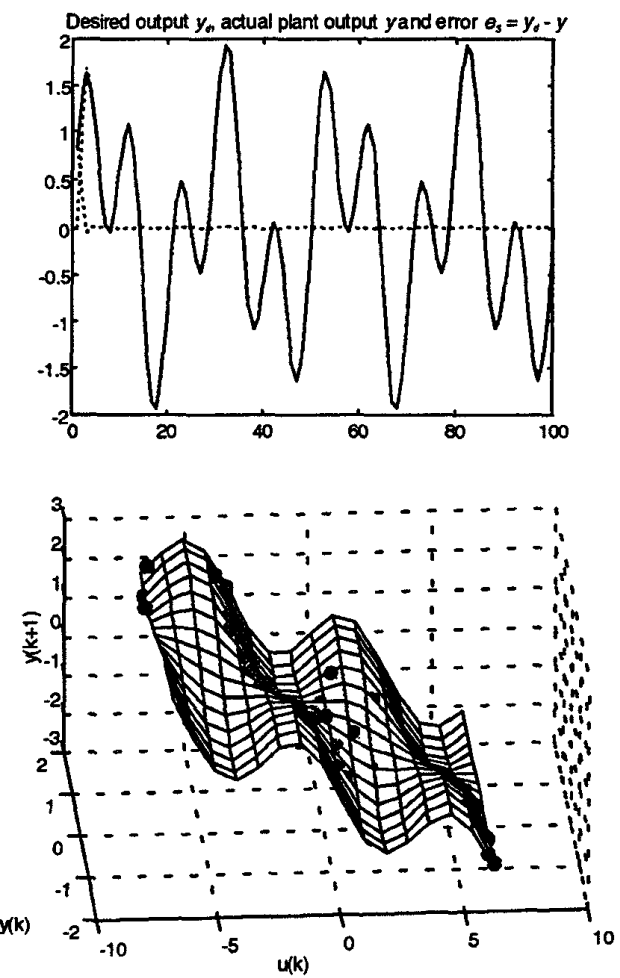

Figure 6 ABC Top: Perfect tracking of the desired signal $y_{d}$ $=\sin (2 \pi k / 25)+\sin (2 \pi k / 10)$ for the time invariant plant $(8)$

Pretrained NN weights are fixed. No adaptation.

Bottom: Trajectory shown by dots lies on the surface described by (8). 

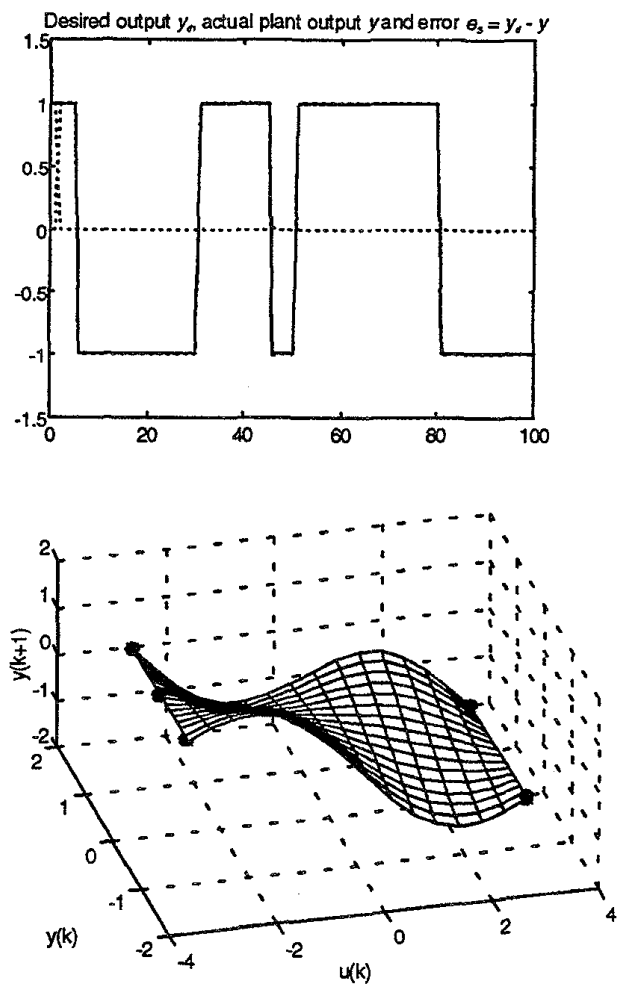

Figure 7 ABC Jop: Perfect tracking of the desired rectangular signal for the time invariant plant (8). Pretrained NN weights are fixed. No adaptation. Bottom: Trajectory shown by dots lies on the surface described by ( 8 ). Desired output $y_{\infty}$ actual plant output $y$ and error $\theta_{s}=y_{d}-y$
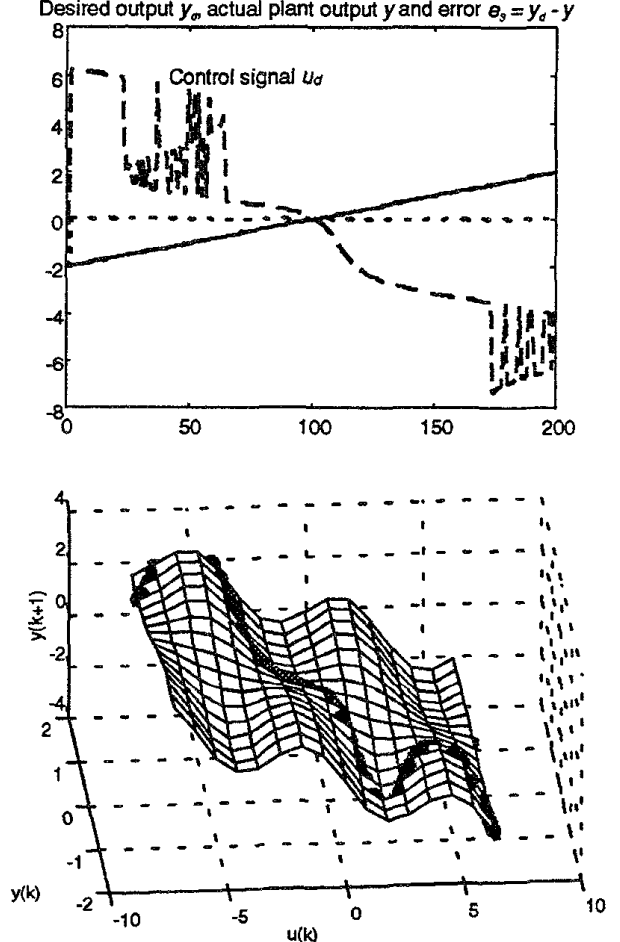

Figure 8 ABC Top: Perfect tracking of the desired ramp signal $[-2,2]$ for the time invariant plant (8) Pretrained NN weights are fixed. No adaptation. Botiom: Trajectory shown by dots lies on or 'sneaks through' the surface described by

(8).

\section{Conclusions}

This paper presents the neural network (or fuzzy logic model) based control of nonlinear dynamic plants. In particular it was shown that the neural network based adaptive backthrough control (ABC) scheme can be successfully applied for control of both linear and nonlinear dynamic plants. We introduced the novel idea of using only one $\mathrm{NN}$, which should simultaneously act as both the plant model and the model of the plant inverse. In this way we avoided a great deal of a redundancy while training two networks. The new learning algorithm based on the NARMAX type of discrete dynamic model is proposed. The $\mathrm{ABC}$ performance seems to be superior to the other NN based adaptive control approaches. For the linear plants, the resulting feedforward controller, providing that the order of the plant and of the plant model are equal, is a perfect adaptive poles-zeros canceller. Thus, the $\mathrm{ABC}$ has a character of a predictive controller. Faced with nonlinear plants the $\mathrm{ABC}$ performs as an nonlinear deadbeat controller. We presented the performance of the $\mathrm{ABC}$ algorithm faced with a much more complex task - with a control of the time variant nonlinear plant. First simulational results seem to be very encouraging.

\section{References}

Agarwal, M., 1997. A systematic Classification of Neural-Network-Based Control, IEEE Control Systems, Vol. 17, No 2, p.p. 75-93

Garcia, C. E. and M. Morari, 1982. Internal Model Control, 1. A Unifying Review and Some New Results, Ind. Eng. Chem. Process Des. Dev. 21, pp. 308-323

Hunt, K. J. and D. Sbarbaro, 1991. Neural networks for nonlinear internal model control, IEE Proc.-D, Vol. 138, No. 5, pp. 431-438

Jordan, M. I., 1993. Connectionist Models of Cognitive Processes, Course 9.641, MTT, Cambridge, MA

Jordan, M. I. and Rumelhart, D. E., 1992. Forward models: Supervised Learning with a Distal Teacher, Journal of Cognitive Science 16, 307-354 
Kecman, V., 1988. Foundations of Automatic Control, (In Serbocroatian), Školska knjiga, Zagreb

Kecman, V. and B.-M., Pfeiffer, 1994. Exploiting the structural equivalence of learning fuzzy systems and radial basis function neural networks, EUFIT '94, Proc., Vol. 1, pp. 58-66, Aachen

Kecman, V., 1997. Neural Networks and Fuzzy Logic Based Control, Report 575, The University of Auckland, Auckland, NZ

Kecman, V. and T. Rommel, 1997. Performance of Neural Networks Based Adaptive Backthrough Control, Proc. of ICONIPANZIIS/ANNES, 1997, Dunedin, New Zealand

Landau, I., 1979. Adaptive Control System: The model reference approach, Marcel Dekker, New York

Narendra, K.S., and K. Parthasarathy, 1990. Identification and control of dynamical systems using neural networks. IEEE Transactions on Neural Networks 1, 4-27

Orr, M. J. L., 1996. Regularization in the selection of radial basis function centers, Report,
Center of Cognitive Science, University of Edinburgh

Psaltis, D., A. Sideris and A. A. Yamamura, 1988. A multilayered Neural Network Controller, IEEE Control System Magazine, 8, pp. 17-21

Rommel, T., 1997. Neural networks based adaptive control, Report No. 97-30 The University of Auckland, Auckland, NZ

Saerens, M., Renders, J. M., H. Bersini, 1996. Neurocontrollers based on backpropagation algorithm, Chap. 7, In IEEE Press Book on Intelligent Control Systems, Gupta $\mathrm{M}$. and $\mathrm{N}$. Sinha (eds.), IEEE Computer

Saerens, M. and A. Soquet, 1991. Neural controllers based on backpropagation algorithm, IEE Proc.-F, 138(1), pp. 55-62

Salman, R. and V. Kecman, 1998. Feedforward Action Based on Adaptive Backthrough Control, Proc. of IPENZ '98, Feb 98, Auckland, NZ

Tsypkin, Ja. Z, 1972. Fundamentals of Automatic Control Theory, (In Russian), Nauka, Moskva Widrow, B. and E., Walach, 1996. Adaptive Inverse Control , Upper Saddle River, NJ, Prentice Hall 